\title{
Optimal Sensor Placement for Model-based Fault Detection and Isolation
}

\author{
Ramon Sarrate, Vicenç Puig, Teresa Escobet and Albert Rosich
}

\begin{abstract}
The problem of optimal sensor placement for FDI consists in determining the set of sensors that minimizes a pre-defined cost function satisfying at the same time a preestablished set of FDI specifications for a given set of faults. The main contribution of this paper is to propose an algorithm for model-based FDI sensor placement based on formulating a mixed integer optimization problem. FDI specifications are translated into constraints of the optimization problem considering that the whole set of ARRs has been generated, under the assumption that all candidate sensors are installed. To show the effectiveness of this approach, an application based on a two-tanks system is proposed.
\end{abstract}

\section{INTRODUCTION}

The sensor placement problem consists in determining the optimal set of sensors to install in a process such that several goals are fulfilled. For instance, observability is a key process property, seek in the design of a process control algorithm. Other desirable properties are reliability, precision, robustness, etc.

There are several articles devoted to the study of the design of sensor networks using goals corresponding to normal monitoring operations. Aside from cost, different other objective functions such as precision [1], reliability [2], or simply observability [1] were used. Different techniques were also used, such as graph theory [2], mathematical programming [3], genetic algorithms [4] and multiobjective optimization [4], among others. The problem has also been extended to incorporate upgrade considerations [5] and maintenance costs [6]. In [7][8], Bagajewicz reviews all these methods and also discusses the applications to bilinear and fully nonlinear systems.

Process disturbances or faults, if undetected, have a serious impact on process economy, product quality, safety, productivity, and pollution level. In order to detect, isolate and correct these abnormal process behaviors, efficient and advanced automated diagnostic systems are of great importance to modern industries. Considerable research has gone into the development of such diagnostic systems [9][10][11]. All model-based approaches for fault detection and isolation in some sense involve the comparison of the observed behavior of the process to a reference model. Process behavior is inferred using sensors measuring the important variables in the process. Hence, the efficiency of the diagnostic approach

This work was supported in part by the Research Comission of the Generalitat of Catalunya (Grup SAC, ref. 2005SGR00537) and by CICYT (ref. DPI-2005-05415) of Spanish Ministry of Education

All authors are with the Automatic Control Department, Universitat Politècnica de Catalunya, Rambla de Sant Nebridi, 10, 08222 Terrassa, Spain ramon.sarrate@upc.edu critically depends on the location of sensors monitoring process variables. The emphasis of most of the work on model-based fault diagnosis has been more on procedures to perform diagnosis given a set of sensors and less on the actual location of sensors for efficient identification of faults.

This paper focuses in the design of a sensor network for model-based Fault Detection and Isolation (FDI) such that faults are detected and eventually isolated. Some contributions have already been done in this direction [12][13][14]. In model-based FDI, faults are modeled as deviations of parameter values or unknown signals and diagnostic models are often brought back to a residual form. Residual quantities are zero in the absence of faults and each residual acts as an alarm that is expected to trigger to a non-zero value upon the occurrence of some faults, in which case the residual is said to be sensitive to these faults. The expected triggering pattern(s) of a set of residuals under some fault is interpreted as the fault signature. Fault isolation is performed by checking the observed residual pattern against different fault signatures [15]. The main approaches to construct residuals are based on using Analytical Redundancy Relations (ARRs) generated either using the parity space [16] or observer approaches [17].

As noticed in [7], the problem of sensor placement in the model-based FDI community is still an open problem. In [13] the sensor placement problem is solved by the analysis of a set of possible ARRs using algorithms of cycle generation in graphs. More recent approaches consist in finding the set of all possible ARRs under the assumption that all possible sensors are installed [14]. Just recently, several exhaustive methods have been developed that claim to generate the complete set of ARRs [18][19][20]. For sensor placement, it is required to use an ARR generation algorithm that is complete. Otherwise, the sensor placement could exclude from consideration some sensor configurations just because some ARRs were not generated. Excluded configurations could provide better FDI results that the ones that were generated. Or, even in some dramatic cases, the sensor placement could not find a solution because of this lack of completeness, whereas, in fact, if all ARRs were generated a solution would have been found.

The main contribution of this paper is to propose an algorithm for model-based FDI sensor placement based on formulating a mixed integer optimization problem. FDI specifications are translated into constraints of the optimization problem considering that the whole set of ARRs has been generated, under the assumption that all candidate sensors are installed. It has been inspired until some extent in [21]. 
There, a mixed-integer linear programming (MILP) formulation for the design of sensor networks for simultaneous process monitoring and fault detection/isolation was presented. The objective was to find a cost-optimal sensor set for a chemical process that provided a good estimate of the state of the system and detected as well as isolated a preestablished set of faults. The optimization problem was casted as an MILP formulation inspired in [3]. The idea was to define a cost function based on a binary optimization vector which stated whether a sensor was installed (1) or not (0). However, constraints were formulated as linear inequalities based on a digraph description of the fault propagation behavior of the process in presence of faults [22]. In the present paper, as already noticed, constraints are formulated on the set of all ARRs generated from the system model considering that the whole set of candidate sensors has been installed. For an alternative MILP formulation of this approach see [23].

The structure of the paper is the following. Section II introduces some model-based FDI basics and states the sensor placement problem for FDI. In Section III, the sensor placement problem is formulated as an optimization problem. Next, Section IV applies this optimal sensor placement approach to a two-tanks system. In Section V, some computation complexity issues are analyzed. Finally, some conclusions and extensions are suggested in Section VI.

\section{MODEL-BASED FDI}

\section{A. The ARR Table}

In model-based FDI, the behavior of a plant is usually modeled by a set of equations, $E$, which in general depend not only on known variables (i.e., measured input and output process variables) but also on unknown variables (i.e., unmeasured internal process variables). In order to evaluate the consistency between the model and measurements taken from available sensors in the process, Analytical Redundancy Relations (ARRs) that only depend on known variables should be generated. ARRs can be obtained by eliminating unknown variables through the convenient manipulation of process equations. For that purpose, structural analysis theory has been extensively used in model-based FDI [9][18][19][20]. A structural model is an abstraction of the equations model, $E$, in which only appears the variables involved in the relations. The structural model can be represented by a binary Incidence Matrix, $M$, which crosses model relations in rows and model variables in columns: an entry $i m_{i j}$ of the matrix is 1 when variable $j$ appears in relation $i$, and 0 otherwise.

According to the structural analysis theory, the binary $A R R$ Table, $A$, crosses measured variables or sensors in columns and all possible ARRs in rows, denoted by $R: a_{i j}=1$ means that ARR $r_{i} \in R$ depends on sensor $s_{j}, a_{i j}=0$ otherwise. For instance, according to Table I, $r_{1}$ only depends on the variables measured by sensors $s_{5}, s_{7}$ and $s_{8}$.

\section{B. The Fault Signature Matrix}

According to the structural analysis approach to FDI [18], each ARR is expected or not to be sensitive to a fault,
TABLE I

EXAMPLE OF AN ARR TABLE

\begin{tabular}{c|cccc} 
& $s_{5}$ & $s_{6}$ & $s_{7}$ & $s_{8}$ \\
\hline$r_{1}$ & 1 & 0 & 1 & 1 \\
$r_{2}$ & 1 & 1 & 0 & 0 \\
$r_{3}$ & 0 & 1 & 1 & 1 \\
$r_{4}$ & 0 & 0 & 0 & 1 \\
$r_{5}$ & 1 & 0 & 1 & 0
\end{tabular}

TABLE II

EXAmple of A FAUlt Signature MATrix

\begin{tabular}{c|cccccccc} 
& $f_{1}$ & $f_{2}$ & $f_{3}$ & $f_{4}$ & $f_{5}$ & $f_{6}$ & $f_{7}$ & $f_{8}$ \\
\hline$r_{1}$ & 0 & 0 & 1 & 0 & 1 & 0 & 1 & 1 \\
$r_{2}$ & 1 & 0 & 0 & 0 & 1 & 1 & 0 & 0 \\
$r_{3}$ & 0 & 1 & 1 & 1 & 0 & 1 & 1 & 1 \\
$r_{4}$ & 1 & 0 & 0 & 0 & 0 & 0 & 0 & 1 \\
$r_{5}$ & 1 & 0 & 0 & 1 & 1 & 0 & 1 & 0
\end{tabular}

characterizing the binary Fault Signature Matrix, M. In this matrix, columns represent faults and rows represent all possible ARRs $R: m_{i k}=1$ means that whenever fault $f_{k}$ occurs, the ARR $r_{i} \in R$ is violated.

Assume that Table II shows the Fault Signature Matrix that corresponds to Table I. According to this table, whenever fault $f_{3}$ is present, ARRs $r_{1}$ and $r_{3}$ are violated.

On a given system, fault detection and isolation properties can be stated based on the information stored by this matrix. Possible properties are:

- Detectability: A set of faults are detectable if their effects on the system can be observed on the available set of ARRs. A fault $f_{k}$ is detectable if at least there is a 1 present in the $k^{\text {th }}$-column of $M$.

- Isolability ${ }^{1}$ : A set of faults are (fully) isolable if their effects can be discriminated one of each other considering the available set of ARRs. Two faults $f_{k}$ and $f_{l}$ are isolable if the $k^{\text {th }}$-column and the $l^{\text {th }}$-column of $M$ are different.

For instance, in Table II all faults are detectable and isolable.

\section{Sensor Placement for Model-Based FDI}

Let $P$ be the set of fixed process components. A tank, a valve, a level sensor and a controller are examples of process components. This set contains the components that are needed for the proper operation of the process, so that the predesigned functional specifications are met. The term 'fixed' denotes that these components are present in any sensor placement configuration. Fixed process components can be affected by faults. Assume that $F_{P}$ is the set of all fixed process components faults.

Let $S$ be the set of candidate sensors. This set contains all possible sensors that can be installed in the system, so that the fault detection and isolation specifications are fulfilled. The term 'candidate' means that the chosen sensor placement

\footnotetext{
${ }^{1}$ Under single-fault isolability assumption
} 
configuration will state which sensors will be present in the process and which not. Let $S^{*} \subseteq S$ denote such set of installed sensors. Assume that every sensor $s_{j} \in S$ can be affected by a fault $f_{j} \in F_{S}$, where $F_{S}$ is the set of candidate sensors faults. Then, $F_{S^{*}}$ will denote the set of installed sensors faults.

As sensors are a kind of process component, let $F=$ $F_{S} \cup F_{P}$ be the set of all possible process components faults. Then, the sensor placement problem for model-based FDI can be stated as follows:

\section{Sensor placement problem for model-based FDI:}

GIVEN a set of candidate sensors, $S$, a structural model, $I M$ (obtained from the set of model equations, E), a Target Fault Set, denoted by $F_{D} \subseteq$ $F$, and a set of model-based FDI specifications, denoted by $T$, FIND a set of installed sensors, $S^{*} \subseteq S$, such that $F_{D}$ fulfils $T$.

Possible model-based FDI specificacions are fault detectability and fault isolability, as stated in the preceding section. The assessment of these specifications for every possible subset of candidate sensors requires the generation of the ARR Table and the Fault Signature Matrix, which must be obtained from $I M$, since both tables are different for each subset.

Given a set of installed sensors, $S^{*} \subseteq S$. Let $A\left(S^{*}\right)$ and $M\left(S^{*}\right)$ denote the ARR Table and the Fault Signature Matrix, with ARRs that just depend on any subset of $S^{*}$. Let $R\left(S^{*}\right)$ be this set of ARRs. A particular case is $\widehat{A}=A(S)$ and $\widehat{M}=M(S)$, denoting the Full ARR Table and the Full Fault Signature Matrix, when all candidate sensors are installed. In this particular case, let $\widehat{R}=R(S)$ denote the Full ARR Set.

Given $\widehat{M}$ and $\widehat{A}$, it is easy to obtain any possible $M\left(S^{*}\right)$. It suffices to eliminate in $\widehat{M}$ the ARRs which depend on sensors $s \in S \backslash S^{*}$, according to $\widehat{A}$. Assume that in Table II $F_{P}=\left\{f_{1}, f_{2}, f_{3}, f_{4}\right\}$ and $F_{S}=\left\{f_{5}, f_{6}, f_{7}, f_{8}\right\}$. Note that for candidate sensor faults the Fault Signature Matrix coincides with the ARR Table. The reason for this is that if an ARR $r_{i}$ depends on a sensor $s_{j}$, then $r_{i}$ is sensible to faults affecting $s_{j}$. If the set of installed sensors is $S^{*}=$ $\left\{s_{5}, s_{7}, s_{8}\right\}$, then $A\left(S^{*}\right)$ just comprises ARRs belonging to the set $R\left(S^{*}\right)=\left\{r_{1}, r_{4}, r_{5}\right\}$. ARRs $r_{2}$ and $r_{3}$ has been discarded since they depend on $s_{6}$, which is not available according to the current configuration, $S^{*}$. Consequently, $M\left(S^{*}\right)$ just comprises ARRs belonging to this set $R\left(S^{*}\right)$. Then, assuming that $F_{D}=\left\{f_{1}, f_{2}, f_{3}, f_{4}\right\}$ and according to the resulting Fault Signature Matrix, faults $f_{1}, f_{3}$ and $f_{4}$ are detectable and isolable, whereas fault $f_{2}$ is not detectable.

Consequently, a possible approach to solve the sensor placement problem for model-based FDI involves that the Full ARR Table and the Full Fault Signature Matrix has already been generated using any of the available complete algorithms [18][19][20]. From these tables, and introducing a cost for each candidate sensor, the sensor placement problem can be translated to an optimization problem, as presented in next section.

\section{OPTIMAL SENSOR PLACEMENT PROBLEM FORMULATION}

\section{A. Optimization Problem Statement}

Let $\mathbf{q}$ be a vector of binary elements that denotes which candidate sensors are installed or not. $q_{j}=1$ means that sensor $s_{j} \in S$ is installed, whereas $q_{j}=0$ means that $s_{j}$ is not. Then, the optimal sensor placement problem can be formulated as the following optimization problem:

$$
\begin{aligned}
& \min : J(\mathbf{q})=\sum_{j=1}^{m} w_{j} q_{j} \\
& \text { subject to } \\
& F_{D} \text { is detectable } \\
& F_{D} \text { is isolable, }
\end{aligned}
$$

where $m$ is the total number of candidate sensors and $w_{j}$ is the cost of sensor $s_{j}$ comprising purchase, maintenance, installation and reliability costs.

Problem (1) will be solved for two general cases:

- CASE I: $F_{D}^{I}=F_{P}$.

- CASE II: $F_{D}^{I I}=F_{P} \cup F_{S^{*}}$

In CASE I, the Target Fault Set is known a priori, before solving the optimization problem. In CASE II, this is not true, since $F_{S^{*}}$ will be known a posteriori, after the optimization problem is solved.

To solve (1), fault detection and isolation specifications must be stated as a set of optimization constraints. Next sections describe how the Full ARR Table and the Full Fault Signature Matrix will serve that purpose.

\section{B. The ARR Selector}

Given a set of installed sensors $S^{*} \subseteq S$, let $\rho_{i}$ be the binary ARR selector denoting whether ARR $r_{i}$ is valid $\left(\rho_{i}=\right.$ $1)$ or not $\left(\rho_{i}=0\right)$, according to $S^{*}$.

The ARR selector can be expressed as in (2), where set $S$ and table $\widehat{A}$ are given, whereas $\mathbf{q}$ is the optimization vector.

$$
\rho_{i}=\prod_{s_{j} \in S}\left[\widehat{A}_{i j} q_{j}+\left(1-\widehat{A}_{i j}\right)\right]
$$

For each candidate sensor $s_{j}$, if $r_{i}$ depends on $s_{j}$, this sensor is required to be installed. If $r_{i}$ does not depend on $s_{j}$, it is not a requirement. Then, $r_{i}$ is valid as long as all required sensors are installed (i.e., they belong to the current sensor placement configuration).

For instance, according to Table I, $\rho_{5}=q_{5} q_{7}$, which means that $r_{5}$ is valid as long as sensors $s_{5}$ and $s_{7}$ are installed.

\section{Fault Detectability Constraint Formulation}

First, CASE I will be considered. The fault detectability requirement can be expressed as (3), where sets $\widehat{R}$ and $F_{P}$ and matrix $\widehat{M}$ are given, and $\rho_{i}$ corresponds to (2).

$$
F_{D}^{I} \text { is detectable } \leftrightarrow \sum_{r_{i} \in \widehat{R}} \widehat{M}_{i k} \rho_{i} \geq 1, \quad \forall f_{k} \in F_{P}
$$


Constraint (3) assures that the column of $\widehat{M}$ which corresponds to fault $f_{k}$ contains at least one 1 associated to a valid ARR.

For instance, given Tables I and II, the fault detectability constraint associated to fault $f_{1}$ is $q_{5} q_{6}+q_{8}+q_{5} q_{7} \geq 1$ and for fault $f_{2}$ is $q_{6} q_{7} q_{8} \geq 1$. So, if the set of installed sensors is $S^{*}=\left\{s_{5}, s_{7}\right\}$, then $f_{1}$ is detectable, whereas $f_{2}$ is not.

In CASE II, faults affecting fixed process components as well as candidate sensors are considered. The constraint formulation (see (4)) depends on the type of fault considered.

$$
\begin{aligned}
& F_{D}^{I I} \text { is detectable } \leftrightarrow \\
& \sum_{r_{i} \in \widehat{R}} \widehat{M}_{i k} \rho_{i} \geq\left\{\begin{array}{ll}
1 & \text { if } f_{k} \in F_{P}, \\
q_{k} & \text { if } f_{k} \in F_{S} .
\end{array} \quad \forall f_{k} \in F\right.
\end{aligned}
$$

According to (4), (3) is applicable whenever a fixed process component fault or a candidate sensor fault is considered, as long as this candidate sensor is installed (i.e., $q_{k}=1$ ). For non-installed candidate sensors, the right hand side of the inequality becomes 0 , meaning that no detectability property is expected for them.

Given Tables I and II, the fault detectability constraint associated to fault $f_{1}$ is $q_{5} q_{6}+q_{8}+q_{5} q_{7} \geq 1$ and for fault $f_{5}$ is $q_{5} q_{7} q_{8}+q_{5} q_{6}+q_{5} q_{7} \geq q_{5}$. So, if the set of installed sensors is $S^{*}=\left\{s_{5}, s_{7}\right\}$, then $f_{1}$ and $f_{5}$ are detectable.

\section{Fault Isolability Constraint Formulation}

First, CASE I will be considered. The fault isolability requirement can be expressed as (5), where sets $\widehat{R}$ and $F_{P}$ and matrix $\widehat{M}$ are given, and $\rho_{i}$ corresponds to (2).

$$
\begin{aligned}
& F_{D}^{I} \text { is isolable } \leftrightarrow \\
& \quad \sum_{r_{i} \in \widehat{R}}\left|\widehat{M}_{i k}-\widehat{M}_{i l}\right| \rho_{i} \geq 1, \forall f_{k}, f_{l} \in F_{P}, f_{k} \neq f_{l}
\end{aligned}
$$

Constraint (5) assures that every two columns of $\widehat{M}$ are different at least in one row associated to a valid ARR.

For instance, given Tables I and II, the fault isolability constraint associated to faults $f_{3}$ and $f_{4}$ is $q_{5} q_{7} q_{8}+q_{5} q_{7} \geq 1$. So, if the set of installed sensors is $S^{*}=\left\{s_{5}, s_{7}\right\}$, then $f_{3}$ and $f_{4}$ are isolable.

Again, the constraint formulation for CASE II (see (6)) depends on the type of fault considered.

$$
\begin{gathered}
F_{D}^{I I} \text { is isolable } \leftrightarrow \\
\sum_{r_{i} \in \widehat{R}}\left|\widehat{M}_{i k}-\widehat{M}_{i l}\right| \rho_{i} \geq \begin{cases}1 & \text { if } f_{k}, f_{l} \in F_{P}, \\
q_{k} & \text { if } f_{l} \in F_{P} \text { and } f_{k} \in F_{S}, \\
q_{l} & \text { if } f_{k} \in F_{P} \text { and } f_{l} \in F_{S}, \\
q_{k} q_{l} & \text { if } f_{k}, f_{l} \in F_{S} .\end{cases} \\
\forall f_{k}, f_{l} \in F, f_{k} \neq f_{l}
\end{gathered}
$$

According to (6), (5) is applicable whenever fixed process component faults or candidate sensor faults are considered, as long as the candidate sensors are installed (i.e., $q_{k}=1$ and $q_{l}=1$ ). For non-installed candidate sensors (either $s_{k}$

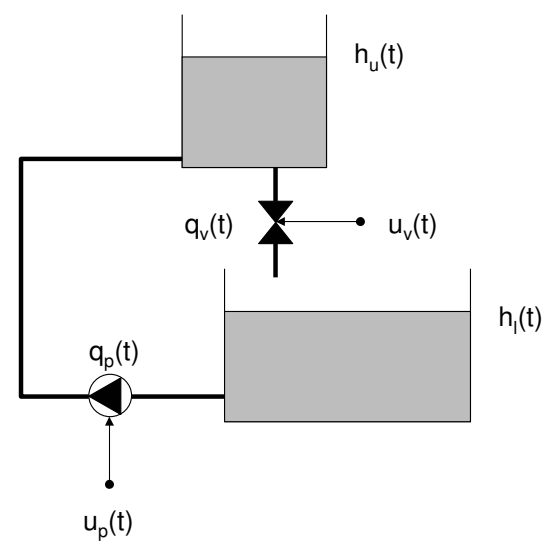

Fig. 1. Two-tanks system

TABLE III

VARIABLES OF THE TWO-TANKS SYSTEM

\begin{tabular}{cc}
\hline Variable & Description \\
\hline$h_{u}$ & upper tank level \\
$h_{l}$ & lower tank level \\
$q_{v}$ & valve flow \\
$q_{p}$ & pump flow \\
$u_{v}$ & valve control input \\
$u_{p}$ & pump control input
\end{tabular}

or $s_{l}$, or both), the right hand side of the inequality becomes 0 , meaning that no isolability property is expected for them.

For instance, given Tables I and II, the fault isolability constraint associated to faults $f_{4}$ and $f_{8}$ is $q_{5} q_{7} q_{8}+q_{8}+$ $q_{5} q_{7} \geq q_{8}$. So, if the set of installed sensors is $S^{*}=\left\{s_{5}, s_{7}\right\}$, then $f_{4}$ and $f_{8}$ are isolable.

\section{APPLICATION TO A TWO-TANKS SYSTEM}

\section{A. Process Description}

The system is made up of two tanks interconnected by a pump and a valve (see Fig. 1). In all, there are four internal variables and two input variables in the system, as summarized in Table III. So the candidate sensor set comprises up to six sensors $S=\left\{h_{u}, h_{l}, q_{v}, q_{p}, u_{v}, u_{p}\right\}$.

Eight hypothetical faults are considered in the system (see Table IV): leaks in the upper and lower tanks, and wrong readings of each candidate sensor. So the fault sets are $F=$ $F_{P} \cup F_{S}=\left\{f_{u}, f_{l}\right\} \cup\left\{f_{h_{u}}, f_{h_{l}}, f_{q_{v}}, f_{q_{p}}, f_{u_{v}}, f_{u_{p}}\right\}$.

TABLE IV

Hypothetical FaUlts of the Two-TANKS System

\begin{tabular}{cc}
\hline Fault & Description \\
\hline$f_{u}$ & upper tank leak \\
$f_{l}$ & lower tank leak \\
$f_{h_{u}}$ & wrong upper tank level sensor reading \\
$f_{h_{l}}$ & wrong lower tank level sensor reading \\
$f_{q_{v}}$ & wrong valve flow sensor reading \\
$f_{q_{p}}$ & wrong pump flow sensor reading \\
$f_{u_{v}}$ & wrong valve control input sensor reading \\
$f_{u_{p}}$ & wrong pump control input sensor reading
\end{tabular}


TABLE V

RESULTS FOR CASE I

\begin{tabular}{|c|c|c|c|c|c|c|c|c|c|}
\hline \multirow{2}{*}{ Sensor } & \multicolumn{7}{|c|}{ Cost distribution 1} & \multicolumn{2}{|c|}{ Cost distr. 2} \\
\hline & Cost & & & alte & nativ & & & Cost & $\mathbf{q}$ \\
\hline$h_{u}$ & 10 & & $\mathrm{X}$ & $X$ & & $\mathrm{X}$ & $\mathrm{X}$ & 100 & \\
\hline$h_{l}$ & 100 & & & $\mathrm{X}$ & $\mathrm{X}$ & & $\mathrm{X}$ & 10 & $\mathrm{X}$ \\
\hline$q_{v}$ & 10 & $X$ & & & $X$ & $X$ & $X$ & 10 & $X$ \\
\hline$q_{p}$ & 10 & $X$ & $X$ & $X$ & $X$ & $X$ & $X$ & 10 & $X$ \\
\hline$u_{v}$ & 10 & $X$ & X & $X$ & $X$ & & & 100 & \\
\hline$u_{p}$ & 100 & $X$ & $X$ & & & $\mathrm{X}$ & & 10 & $X$ \\
\hline
\end{tabular}

TABLE VI

RESUlTS FOR CASE II

\begin{tabular}{|c|c|c|c|c|c|c|c|c|c|}
\hline \multirow{4}{*}{$\begin{array}{c}\text { Sensor } \\
h_{u} \\
h_{l}\end{array}$} & \multicolumn{5}{|c|}{ Cost distribution 1} & \multicolumn{4}{|c|}{ Cost distribution 2} \\
\hline & \multirow{2}{*}{$\begin{array}{c}\text { Cost } \\
10\end{array}$} & \multicolumn{4}{|c|}{ q alternative } & \multirow{2}{*}{$\begin{array}{c}\text { Cost } \\
100\end{array}$} & \multicolumn{3}{|c|}{ q alternative } \\
\hline & & & & $\mathrm{X}$ & $\mathrm{X}$ & & & & $\mathrm{X}$ \\
\hline & 100 & $\mathrm{X}$ & $\mathrm{X}$ & $\mathrm{X}$ & $\mathrm{X}$ & 10 & $\mathrm{X}$ & $\mathrm{X}$ & $\mathrm{X}$ \\
\hline$q_{v}$ & 10 & $\mathrm{X}$ & & & $\mathrm{X}$ & 10 & $\mathrm{X}$ & & $\mathrm{X}$ \\
\hline$q_{p}$ & 10 & & $\mathrm{X}$ & & & 10 & & $\mathrm{X}$ & \\
\hline$u_{v}$ & 10 & $\mathrm{X}$ & $\mathrm{X}$ & $\mathrm{X}$ & & 100 & $\mathrm{X}$ & $\mathrm{X}$ & \\
\hline$u_{p}$ & 100 & $\mathrm{X}$ & $\mathrm{X}$ & $\mathrm{X}$ & $\mathrm{X}$ & 10 & $\mathrm{X}$ & $\mathrm{X}$ & $\mathrm{X}$ \\
\hline
\end{tabular}

\section{B. Optimization results}

Applying the exhaustive ARR generation algorithm described in [19] a Full ARR Table and a Full Fault Signature Matrix was created, comprising up to 35 ARRs.

Problem (1) along with constraints (3) and (5) corresponds to CASE I. This optimization problem was implemented in ILOG OPL Studio [24], involving 3 constraints (i.e., 2 regarding the detectability specification and 1 for the isolability specification). Results are given in Table $\mathrm{V}$ for different candidate sensors cost distributions. A ' $X$ ' in the table indicates that the corresponding sensor is installed.

Cost distribution 1 suggested six alternative sensor configurations with the same minimum total cost of 130 , whereas cost distribution 2 produced just a unique solution, with a minimum global cost of 40 . Other cost distributions were tested and the optimization algorithm always suggested a sensor configuration of 4 sensors.

Problem (1) along with constraints (4) and (6) corresponds to CASE II. This optimization problem was also implemented in ILOG OPL Studio, now involving 36 constraints (i.e., 8 regarding the detectability specification and 28 the isolability specification). Results are given in Table VI for different candidate sensors cost distributions.

In this case, cost distribution 1 suggested just four alternative sensor configurations with a minimum total cost of 220 . Despite its higher cost, all these alternatives included sensors $h_{l}$ and $u_{p}$. Thus, these sensors will be part of all solutions, no matter what cost is assigned to them. In cost distribution 2, three alternatives where given; all included both sensors.

Again, other cost distributions were tested and the optimization algorithm always suggested a sensor configuration of 4 sensors. So, for the two-tank system, the cardinality of $S^{*}$ will always be 4 .

Notice that for CASE I the optimization algorithm produced cheaper solutions than for CASE II. This was expected,
TABLE VII

Simplified FAUlt Signature MAtriX FOR $S^{*}=\left\{h_{l}, q_{v}, u_{p}, u_{v}\right\}$

\begin{tabular}{c|cccccc} 
& $f_{u}$ & $f_{l}$ & $f_{h_{l}}$ & $f_{q_{v}}$ & $f_{u_{p}}$ & $f_{u_{v}}$ \\
\hline$r_{9}$ & 0 & 1 & 1 & 1 & 1 & 1 \\
$r_{18}$ & 1 & 0 & 1 & 1 & 1 & 1 \\
$r_{23}$ & 1 & 1 & 1 & 1 & 0 & 1 \\
$r_{27}$ & 1 & 1 & 1 & 1 & 1 & 0 \\
$r_{32}$ & 1 & 1 & 0 & 1 & 1 & 1 \\
$r_{34}$ & 1 & 1 & 1 & 0 & 1 & 1
\end{tabular}

since constraints in CASE I are more relaxed (i.e., a solution to CASE I is more easily attainable than for CASE II).

In order to verify that the optimization algorithm is suggesting feasible solutions to the FDI specifications, Table VII shows the simplified fault signature matrix corresponding to the first sensor configuration alternative given in Table VI for cost distribution 1. This simplified fault signature matrix has been obtained by eliminating, in the Full Fault Signature Matrix, the columns that correspond to faults $f_{h_{u}}$ and $f_{q_{p}}$, that affect sensors not installed, and the rows that correspond to ARRs that depend on these sensors not installed. Every column in Table VII contains at least one 1 , and all columns are different. Thus, sensor configuration $S^{*}=\left\{h_{l}, q_{v}, u_{p}, u_{v}\right\}$ satisfies the detectability and isolability specifications.

\section{COMPUTATIONAL COMPLEXITY ISSUES}

Finding a solution to problem (1) is not trivial because of its combinatorial nature. As it is known, combinatorial problems fall in the NP category with a complexity that depends exponentially with the number of optimization variables. In particular, solving time for problem (1) clearly depends on the number and complexity of the optimization constraints, which in turn, according to section III, depend on the sizes of the Full ARR Table and the Full Fault Signature Matrix.

On the one hand, for CASE II the number of constraints, $n_{C}$, depends on the number of faults, $\operatorname{card}(F)$ (see (7)). There is a fault detectability constraint for each fault in $F$ (see (4)) and a fault isolability constraint for every possible combination of two faults out of $F$ (see (6)).

$$
\begin{aligned}
& n_{C}=\left.n_{C}\right|_{\text {detectability }}+\left.n_{C}\right|_{\text {isolability }} \\
= & \operatorname{card}(F)+\left(\begin{array}{c}
\operatorname{card}(F) \\
2
\end{array}\right)=\frac{\operatorname{card}(F)(\operatorname{card}(F)+1)}{2}
\end{aligned}
$$

On the other hand, the complexity of the constraints depends on the number of candidate sensors, $\operatorname{card}(S)$ (see (2)), and the number of all possible ARRs, $\operatorname{card}(\widehat{R})$ (see (3)-(6)).

In the previous section, an application to a simple twotanks system has been presented. In this case, computational complexity was not a real concern. In order to see the limitations of the proposed sensor placement method, a more demanding application was used (see [25]), involving 17 faults and 8 candidate sensors, which, according to (7), posed an optimization problem with up to 153 constraints. 
TABLE VIII

Computational CompleXity STUdy

\begin{tabular}{cc}
\hline nr. of ARRs & solving time (secs.) \\
\hline 126 & 7.25 \\
169 & 9.88 \\
410 & 47.63 \\
701 & 159.06 \\
1135 & 691.77 \\
9029 & $>6$ hours
\end{tabular}

Several cases were analyzed corresponding to different subsets of the Full ARR Set, whose total size was 9029. The optimal sensor placement problem was solved for increasing number of ARRs (i.e., increasing sizes of the Full ARR Table and the Full Fault Signature Matrix were considered). Table VIII, illustrates the exponential dependance of the solving time $^{2}$ on the number of the ARRs being involved.

These results show the main drawback of the sensor placement method presented in this paper, which clearly limits at present its applicability to complex systems.

\section{CONCLUSIONS}

This paper has addressed the problem of optimal sensor placement for Model-based FDI. This problem consists in determining the set of sensors that minimizes a pre-defined cost function satisfying at the same time a pre-established set of FDI specifications for a given set of faults. The main contribution of this paper has been to propose an algorithm for model-based FDI sensor placement based on formulating a mixed integer optimization problem. Any FDI specification could be taken into account as long as it could be translated to a constraint of the optimization problem. Fault detectability and isolability constraints have been formulated in this paper, but other specifications such as fault identifiability, fault sensitivity, etc., could be easily included in the optimal sensor placement problem. To show the effectiveness of this approach, an application based on a two-tanks system has been proposed.

However, this approach presents some drawbacks that should be addressed in further research. First, constraints are non-linear. That leads to a mixed-integer non-linear problem that in general has a high computational complexity. Second, the method requires a Full ARR Table and a Full Fault Signature Matrix, that can be obtained considering the system model structure and all candidate sensors installed. The size of these tables grows exponentially with the number of sensors considered. A possible way to get around these problems is to develop an algorithm that tries to avoid the computational burden of the approach proposed in this paper by constructing incrementally the optimal set of sensors to be installed in order to fulfill the FDI requirements [25].

\section{REFERENCES}

[1] M. Luong, D. Maquin, C. T. Huynh, and J. Ragçot, "Observability, redundancy, reliability and integrated design of measurement systems,"

${ }^{2}$ On a HP Compaq nx5000 notebook, Pentium M 1500 MHz, Win XP in Proc. $2^{\text {nd }}$ IFAC Symposium on Intelligent Components and Instrument Control Applications, Budapest, Hungary, 1994.

[2] Y. Ali and S. Narasimhan, "Sensor network design for maximizing reliability of linear processes," AIChE J., vol. 39, no. 5, pp. 820-828, 1993.

[3] M. Bagajewicz and E. Cabrera, "A new MILP formulation for instrumentation network design and upgrade," in Proc. $4^{\text {th }}$ IFAC Workshop on On-Line Fault Detection and Supervision in the Chemical Process Industries, Seoul, Korea, June 8-9, 2001.

[4] A. Viswanath and S. Narasimhan, "Multi-objective sensor network design using genetic algorithms," in Proc. $4^{\text {th }}$ IFAC Workshop on On-Line Fault Detection and Supervision in the Chemical Process Industries, Seoul, Korea, June 8-9, 2001.

[5] M. Bagajewicz and M. Sánchez, "Reallocation and upgrade of instrumentation process plants," Comput. Chem. Eng., vol. 24, no. 8, pp. 1945-1959, 2000.

[6] M. Sánchez and M. Bagajewicz, "On the impact of corrective maintenance in the design of sensor networks," Ind. Eng. Chem. Res., vol. 39, no. 4, pp. 977-981, 2000.

[7] M. Bagajewicz, Design and Upgrade of Process Plant Instrumentation. Lancaster, PA: Technomic Publishers, 2000.

[8] - "Review of recent results in instrumentation design and upgrade for process plants," in Proc. $4^{\text {th }}$ IFAC Workshop on On-Line Fault Detection and Supervision in the Chemical Process Industries, Seoul, Korea, June 8-9, 2001.

[9] M. Blanke, M. Kinnaert, J. Lunze, and M. Staroswiecki, Diagnosis and Fault-Tolerant Control. Springer, 2003.

[10] J. Gertler, Fault Detection and Diagnosis in Engineering Systems. New York: Marcel Dekker, 1998.

[11] J. Chen and R. Patton, Robust Model-Based Fault Diagnosis for Dynamic Systems. Boston: Kluwer Academic Publishers, 1999.

[12] F. Nejjari, R. Pérez, T. Escobet, and L. Travé-Massuyès, "Fault diagnosability utilizing quasi-static and structural modelling," Math. Comput. Mod., vol. 45, pp. 606-616, 2006.

[13] D. Maquin, M. Luong, and J. Ragot, "Fault detection and isolation and sensor network design," Europ. J. Autom., vol. 31, no. 13, pp. 396-406, 1997.

[14] S. Spanache, T. Escobet, and L. Travé-Massuyès, "Sensor placement optimisation using genetic algorithms," in Proc. $15^{\text {th }}$ International Workshop on Principles of Diagnosis (DX'04), Carcassonne, France, June 23-25, 2004.

[15] J. Gertler, "Survey on model based failure detection and isolation in complex plants," IEEE Control Syst. Mag., vol. 8, no. 6, pp. 3-11, 1988.

[16] M. Staroswiecki and G. Comtet-Varga, "Analytical redundancy relations for fault detection and isolation in algebraic dynamic systems," Automatica, vol. 37, no. 5, pp. 687-699, 2001.

[17] R. Nikoukhah, "A new methodology for observer design and implementation," IEEE Trans. Automat. Contr., vol. 43, no. 2, pp. 229-234, 1998.

[18] D. Düştegör, E. Frisk, V. Cocquempot, M. Krysander, and M. Staroswiecki, "Structural analysis of fault isolability in the DAMADICS benchmark," Contr. Eng. Pract., vol. 14, pp. 597-608, 2006.

[19] M. Krysander, "Design and analysis of diagnosis systems using structural analysis," Ph.D. dissertation, Linköping Univ., Linköping, Sweden, June 2006.

[20] L. Travé-Massuyès, T. Escobet, and X. Olive, "Diagnosability analysis based on component supported analytical redundancy relations," IEEE Trans. Syst., Man, Cybern. A, vol. 36, no. 6, pp. 1146-1160, 2006.

[21] M. Bagajewicz, A. Fuxman, and A. Uribe, "Instrumentation network design and upgrade for process monitoring and fault detection," AIChE J., vol. 50, no. 8, pp. 1870-1880, Aug. 2004.

[22] R. Raghuraj, M. Bhushan, and R. Rengaswamy, "Locating sensors in complex chemical plants based on fault diagnostic observability criteria," AIChE J., vol. 45, no. 2, pp. 310-322, Feb. 1999.

[23] A. Fijany and F. Vatan, "A new efficient algorithm for analyzing and optimizing the system of sensors," in Proc. 2006 IEEE Aerospace Conference, Big Sky, Montana, USA, Mar. 4-11, 2006.

[24] The ILOG website. [Online]. Available: http://www.ilog.com/

[25] A. Rosich, R. Sarrate, V. Puig, and T. Escobet, "Efficient optimal sensor placement for model-based FDI using and incremental algorithm," in Proc. $46^{\text {th }}$ IEEE Conference on Decision and Control, New Orleans, USA, Dec. 12-14, 2007. 